\title{
Eficácia de herbicidas em condições controladas para o controle de gramíneas infestantes de canaviais em estiagem ${ }^{1}$
}

\author{
Efficacy of herbicides in controlled conditions for controlling sugarcane grass \\ weeds under water restriction
}

\begin{abstract}
Paulo Roberto Fidelis Giancotti ${ }^{2}$; Roberto Estêvão Bragion de Toledo ${ }^{3}$; Pedro Luís da Costa Aguiar Alves ${ }^{4}$; Ricardo Victoria Filho ${ }^{5}$; Eduardo Negrisoli ${ }^{6}$; João Batista Cason ${ }^{3}$; Samuel Neves Rodrigues Alves ${ }^{3}$; Murilo Grespan da Rocha ${ }^{7}$
\end{abstract}

Resumo - O controle químico das plantas daninhas em canaviais pode ser realizado no período de estiagem devido, principalmente, planejamento de operações agrícolas, e logística quanto ao uso de equipamentos e mão-de-obra. $\mathrm{O}$ presente estudo teve como objetivo avaliar a eficácia agronômica de herbicidas para o controle de plantas daninhas gramíneas infestantes de canaviais, utilizando diferentes períodos de restrição hídrica em condições controladas. As plantas daninhas avaliadas foram Digitaria nuda, Panicum maximum e Brachiaria decumbens. Os tratamentos herbicidas aplicados em pré-emergência total das plantas daninhas foram: diuron + hexazinone + sulfometuron-methyl $(1387+391+33,35 ; 1507,5+425+36,25 ; 1658,25+467,5+39,87$ e $1809+510+43,5$ g i.a. ha $\left.{ }^{-1}\right)$; amicarbazone $\left(1190\right.$ g i.a. ha $\left.{ }^{-1}\right)$; amicarbazone + isoxaflutole $(840$ $+82,5$ g i.a. ha $^{-1}$ ) e uma testemunha sem aplicação. Após a aplicação, as parcelas foram submetidas a 0, 30, 60 e 90 dias de restrição hídrica. Foram realizadas avaliações visuais de controle e aos 28 dias após o restabelecimento da umidade, determinada a biomassa das plantas daninhas. Todas as doses de diuron + hexazinone + sulfometuron-methyl foram eficazes no controle das espécies estudadas em todos os períodos de restrição hídrica. Amicarbazone aplicado isoladamente foi ineficiente no controle das plantas daninhas sob a condição de 60 dias de seca. Amicarbazone + isoxaflutole, apresentou bom controle das plantas daninhas, com exceção de Digitaria nuda, quando na condição a 60 dias de seca.

Palavras-chaves: época seca, pré-emergência, cana-de-açúcar, capim-colonião, capim-colchão, capim-braquiária

Abstract - Chemical control of weeds in sugarcane crop may be performed in dry season, mainly due to agricultural operations planning, and logistics in relation to the use of equipment and labor. This study aimed to evaluate the agronomic efficacy of herbicides for controlling sugarcane grass weeds by using different periods of water restriction. Evaluated weeds were

\footnotetext{
${ }^{1}$ Recebido para publicação em 30/01/2013 e aceito em 28/05/2013.

${ }^{2}$ Doutorando do programa de Pós-graduação em Produção Vegetal da Faculdade de Ciências Agrárias e Veterinárias (FCAV/UNESP), Jaboticabal, SP. Brasil. Email: <paulogiancotti@gmail.com>. (Autor para correspondência).

${ }^{3}$ Pesquisador da DuPont do Brasil.

${ }^{4}$ Professor Adjunto da Faculdade de Ciências Agrárias e Veterinárias (FCAV/UNESP).

${ }^{5}$ Professor Titular da Escola Superior de Agricultura "Luiz de Queiroz" (ESALQ - USP).

${ }^{6}$ Pesquisador na TechField Assessoria e Consultoria Agrícola e Ambiental.

${ }^{7}$ Pesquisador na Pioneer Sementes (DuPont do Brasil).
} 
Digitaria nuda, Panicum maximum and Brachiaria decumbens. Herbicides treatments applied in total pre-emergency condition were: diuron + hexazinone + sulfometuron-methyl $(1387+391+$ $33.35 ; 1507.5+425+36.251658 .25+467.5+39.87$ and $1809+510+43.5 \mathrm{~g} \mathrm{a.i.} \mathrm{ha}{ }^{-1}$; amicarbazone (1190 g a.i. ha $\left.{ }^{-1}\right)$; amicarbazone + isoxaflutole $\left(840+82.5 \mathrm{~g}\right.$ a.i. ha $\left.{ }^{-1}\right)$ and an check without application. After application, plots were submitted to 0, 30, 60 and 90 days of water restriction. Control visual evaluations were performed 28 days after humidity restoration, with the weeds biomass determination. All studied doses of diuron + hexazinone + sulfometuronmethyl were effective in controlling the plants studied in all water restriction periods. Amicarbazone sprayed alone was not efficient on weeds control under the condition of 60 days with water restriction. Amicarbazone + isoxaflutole presented satisfactory weeds control, except for Digitaria nuda, in 60 days of water restriction.

Keywords: dry season, pre-emergent, sugarcane, crabgrass, guinea grass, surinam grass

\section{Introdução}

O controle químico é o principal método de controle das plantas daninhas em áreas de cana-de-açúcar, sendo que existem diferentes herbicidas seletivos e com espectro de ação sobre diferentes alvos. Entretanto, independentemente da comunidade infestante presente, os herbicidas necessitam de umidade no solo para que as suas moléculas fiquem disponíveis em sua solução, podendo assim ser absorvidas pelas plantas daninhas (Martini \& Durigan, 2004).

O manejo químico é mais eficaz quando realizado durante a estação das águas, pois a umidade no solo e o intenso metabolismo das plantas daninhas favorecem a absorção da maior parte dos herbicidas registrados para a cultura da cana-de-açúcar, os quais apresentam solubilidade baixa em água. No entanto, diversas pesquisas relatam bons resultados de controle em períodos secos com o uso dos herbicidas amicarbazone, imazapic, isoxaflutole, tebuthiuron e diuron + hexazinone + sulfometuron, o que pode ser explicado pelo fato de apresentam características físicoquímicas favoráveis, como alta solubilidade em água e baixa capacidade de adsorção nos minerais de argila e matéria orgânica do solo (Negrisoli et al., 2011; Toledo et al., 2010; Correia et al., 2010; Carbonari et al., 2009; Rodrigues \& Almeida, 2011).

$\mathrm{O}$ uso de herbicidas em pré-emergência das plantas daninhas tem-se destacado na cultura da cana-de-açúcar, pois proporciona melhor posicionamento e distribuição do produto químico sobre o alvo, a manutenção da cultura no limpo e a otimização de maquinário e mão-de-obra (Martini \& Durigan, 2004). A persistência desses produtos no solo varia de acordo com a estrutura química da molécula, o tipo de solo e as condições climáticas, como a sua umidade, que afetam por sua vez a adsorção, lixiviação e a decomposição microbiana e química (Silva et al., 1999).

Entre os herbicidas recomendados para a época seca e registrados para a cultura de cana-de-açúcar, encontra-se amicarbazone, isoxaflutole e a associação de diuron + hexazinone + sulfometuron-methyl. A maioria desses herbicidas apresenta ação em pré e pósemergência inicial, sendo recomendados para controle de espécies de plantas daninhas gramíneas, folhas largas e perenes de difícil controle (Procópio et al., 2004).

Pertencente ao grupo químico das triazolinonas, o amicarbazone é um herbicida com alta solubilidade e baixa adsorção, sendo recomendado para aplicação em épocas secas. O amicarbazone tem como mecanismo de ação a inibição do fluxo de elétrons no fotossistema II, e por isso, promove sintomas de clorose, redução de crescimento e necrose foliar, nas plantas suscetíveis (Toledo, 2004).

$\mathrm{O}$ isoxaflutole apresenta boa estabilidade em condições de seca, mantendo efeito residual, dependendo da dose, por mais 
de 60 dias após o início das chuvas (Rodrigues e Almeida, 2011).

O sulfometuron-methyl, é um inibidor da enzima ALS (acetolactato sintase), e amplamente utilizado como maturador em cana-de-açúcar. Recentemente, também tem sido comercializado como herbicida para a cultura, quando formulado em mistura pronta juntamente com diuron e hexazinone. A utilização simultânea de dois ou mais herbicidas sobre uma mesma cultura vem sendo cada vez mais empregada na agricultura e representa um avanço nas estratégias de controle sobre as plantas daninhas resistentes e também de difícil controle, além do racionamento das operações de tratos culturais (Kruse, 2001). O sinergismo que ocorre quando se associam dois ou mais herbicidas e ocorre ação de complementaridade entre os mesmos, é a resposta de um produto facilitando a ação física e ou bioquímica do outro (Matthews, 1994).

O diuron é um herbicida não-ionizável, relativamente persistente em solos (meia-vida de dissipação de 90 a 180 dias), sendo que a principal forma de dissipação é a degradação microbiológica. Assim como o diuron, o hexazinone é um herbicida inibidor do fluxo de elétrons no fotossistema II, mas que apresenta alta solubilidade, sendo mais recomendado para a época seca (Rodrigues \& Almeida, 2011). Em canaviais, principalmente nos quais a queimada para a colheita ainda é uma realidade, as invasoras classificadas como gramíneas podem causar elevada redução na produtividade da cultura.

A associação de ingredientes ativos com diferentes características convenientes ao período de restrição hídrica pode, através de uma ação de sinergismo, controlar as plantas daninhas gramíneas de uma forma superior. $\mathrm{O}$ objetivo do estudo foi determinar o tratamento químico mais eficaz para o controle de plantas daninhas gramíneas, infestantes de canaviais, em diferentes períodos de restrição hídrica.

\section{Material e Métodos}

O experimento foi conduzido em casade-vegetação, na área experimental do Departamento de Biologia Aplicada à Agropecuária, da Faculdade de Ciências Agrárias e Veterinárias de Jaboticabal (FCAV - UNESP).

Os tratamentos foram dispostos segundo o delineamento experimental inteiramente casualizado, em esquema fatorial 4 x 7, sendo quatro períodos de restrição hídrica e sete tratamentos químicos, com três repetições. Os tratamentos químicos foram representados pelos herbicidas: diuron + hexazinone + sulfometuron-methyl (1387+ $391+33,35 ; 1507,5+425+36,25 ; 1658,25+$ $467,5+39,87$ e $1809+510+43,5$ g i.a. ha $\left.{ }^{-1}\right)$ amicarbazone (1190 g i.a. ha ${ }^{-1}$ ), amicarbazone + isoxaflutole $\left(840+82,5\right.$ g i.a. ha $\left.{ }^{-1}\right)$ e testemunha sem aplicação. Cada parcela constituiu-se de uma caixa com capacidade de $15 \mathrm{~L}$ de solo, nas quais foram semeadas três espécies de plantas infestantes em canaviais paulistas: Digitaria nuda Shumach, Panicum maximum Jacq.e Brachiaria decumbens Stapf (código DIGSS, PANMA e BRADC respectivamente). O solo utilizado foi um Latossolo vermelho distrófico, retirado de uma camada arável, em Jaboticabal- SP. O solo apresentava as seguintes características físicas: $54,4 \%$ de areia total, $35,9 \%$ de argila e $9,7 \%$ de silte.

A aplicação dos herbicidas foi realizada por meio de um equipamento costal pressurizado a $\mathrm{CO}_{2}$, munido de barra com seis pontas XR 110.02 e regulado para utilizar volume de calda de $200 \mathrm{~L} \mathrm{ha}^{-1}$. Após a aplicação dos herbicidas as parcelas foram submetidas a diferentes períodos de restrição hídrica e protegidas da chuva dentro da casade-vegetação por períodos de $0,30,60$ e 90 dias de restrição hídrica (DRH).

As avaliações visuais de controle das plantas daninhas ocorreram aos 7, 14, 21 e 28 dias após o restabelecimento da umidade (DARU), utilizando-se escala de notas 
variando de 0 a $100 \%$, sendo $0 \%$ representou nenhuma injúria e $100 \%$ a morte das plantas. Após a última avaliação (28 DARU), a parte aérea das plantas foram cortadas rente ao solo, separadas por espécie e períodos de restrição hídrica, e acondicionadas em estufa com ventilação forçada de ar, a $70{ }^{\circ} \mathrm{C}$, até atingir massa constante. Em seguida, o material foi pesado em balança eletrônica de precisão de $0,0001 \mathrm{~g}$.

Os dados foram submetidos à análise de variância e quando detectadas diferenças significativas entre os tratamentos, suas médias foram comparadas pelo teste de Tukey a 5\% de probabilidade.

\section{Resultados e Discussão}

A B. decumbens, apesar de ser considerada uma planta daninha de difícil controle, foi totalmente controlada por todos os tratamentos químicos e períodos de restrição hídrica, ao final de 28 DARU; com exceção apenas do amicarbazone isolado, submetido a 60 DRH (Tabela 1). Dos 14 aos 28 DARU, o amicarbazone apresentou o controle reduzido sobre essa espécie, diferindo dos demais. $B$. decumbens tratada com amicarbazone isolado submetido a $60 \mathrm{DRH}$ atingiu uma biomassa de $81 \%$ em relação à testemunha, ou seja, nessa situação, o controle foi insuficiente.

Tabela 1. Controle visual (\%) de Brachiaria decumbens (BRADC) submetidas a 0, 30, 60 e 90 dias de restrição hídrica (DRH), aos 7, 14, 21 e 28 dias após o restabelecimento da umidade (DARU). Jaboticabal, SP. 2011.

\begin{tabular}{|c|c|c|c|c|c|c|c|c|c|}
\hline \multirow{2}{*}{ Tratamentos } & \multirow{2}{*}{ Dose (g i.a. ha-1) } & \multicolumn{4}{|c|}{ Controle de BRADC aos 7 DARU } & \multicolumn{4}{|c|}{ Controle de BRADC aos 14 DARU } \\
\hline & & $0 \mathrm{DRH}$ & $30 \mathrm{DRH}$ & $60 \mathrm{DRH}$ & $90 \mathrm{DRH}$ & $0 \mathrm{DRH}$ & $30 \mathrm{DRH}$ & $60 \mathrm{DRH}$ & $90 \mathrm{DRH}$ \\
\hline Diu + Hex + Sulfom ${ }^{1}$ & $87+391+33,35$ & $40,0 \mathrm{ABb}$ & $63,3 \mathrm{Aab}$ & $46,7 \mathrm{ABab}$ & $85,0 \mathrm{Aa}$ & $98,3 \mathrm{Aa}$ & $99,7 \mathrm{Aa}$ & $86,7 \mathrm{Aa}$ & $100,0 \mathrm{Aa}$ \\
\hline $\mathrm{Diu}+\mathrm{Hex}+$ Sulfom & $1507,5+425+36,25$ & $81,7 \mathrm{Aa}$ & & $73,3 \mathrm{Aba}$ & $97,0 \mathrm{Aa}$ & $100,0 \mathrm{Aa}$ & & $93,3 \mathrm{Aa}$ & $100,0 \mathrm{Aa}$ \\
\hline $\mathrm{Diu}+\mathrm{Hex}+$ Sulfom & $1658,25+467,5+39,87$ & $61,7 \mathrm{ABab}$ & $66,7 \mathrm{Aab}$ & $41,7 \mathrm{Bb}$ & $97,5 \mathrm{Ab}$ & $99,3 \mathrm{Aa}$ & $98,3 \mathrm{Aa}$ & $100,0 \mathrm{Aa}$ & $100,0 \mathrm{Aa}$ \\
\hline $\mathrm{Diu}+\mathrm{Hex}+$ Sulfom & $1809+510+43,5$ & $53,3 \mathrm{ABbc}$ & & $40,0 \mathrm{Bc}$ & $90,0 \mathrm{Aab}$ & $98,7 \mathrm{Aa}$ & $100,0 \mathrm{Aa}$ & $100,0 \mathrm{Aa}$ & $100,0 \mathrm{Aa}$ \\
\hline Amicarbazone & 1190 & $30,0 \mathrm{Bb}$ & $50,0 \mathrm{Aab}$ & $36,7 \mathrm{Bb}$ & $87,5 \mathrm{Aa}$ & $100,0 \mathrm{Aa}$ & $90,0 \mathrm{Aa}$ & $56,7 \mathrm{Bb}$ & $95,0 \mathrm{Aa}$ \\
\hline \multirow[t]{3}{*}{ Amicarb+Isoxafl ${ }^{2}$} & $840+82,5$ & $56,7 \mathrm{Aba}$ & $90,0 \mathrm{Aa}$ & $88,3 \mathrm{Aa}$ & $96,0 \mathrm{Aa}$ & $100,0 \mathrm{Aa}$ & $100,0 \mathrm{Aa}$ & $93,3 \mathrm{Aa}$ & $100,0 \mathrm{Aa}$ \\
\hline & & C.V.(\%) & 27,43 & & $5,08^{* *}$ & C.V. $(\%)$ & 10,07 & F trat & $3,67 * *$ \\
\hline & & F trat $\mathrm{x}$ époc & $1,43 \mathrm{~ns}$ & F époc & $16,89 * *$ & F trat $x$ époc & $1,94^{*}$ & F époc & $5,18^{* * *}$ \\
\hline \multirow{2}{*}{ Tratamentos } & \multirow{2}{*}{ Dose (g i.a. ha-1) } & \multicolumn{4}{|c|}{ Controle de BRADC aos 21 DARU } & \multicolumn{4}{|c|}{ Controle de BRADC aos 28 DARU } \\
\hline & & $0 \mathrm{DRH}$ & $30 \mathrm{DRH}$ & $60 \mathrm{DRH}$ & $90 \mathrm{DRH}$ & $0 \mathrm{DRH}$ & $30 \mathrm{DRH}$ & $60 \mathrm{DRH}$ & $90 \mathrm{DRH}$ \\
\hline $\mathrm{Diu}+\mathrm{Hex}^{+}$ & & $98,7 \mathrm{Aa}$ & & & $100,0 \mathrm{Aa}$ & $100,0 \mathrm{Aa}$ & & $\overline{\mathrm{Aa}}$ & \\
\hline & $1507,5+425+36,25$ & $99,0 \mathrm{Aa}$ & & & $100,0 \mathrm{Aa}$ & $100,0 \mathrm{Aa}$ & & $0 \mathrm{Aa}$ &, $0 \mathrm{Aa}$ \\
\hline Diu+Hex +Sulfom & $1658,25+467,5+39,87$ & $99,0 \mathrm{Aa}$ & $0,0 \mathrm{Aa}$ & $100,0 \mathrm{Aa}$ & $100,0 \mathrm{Aa}$ & $100,0 \mathrm{Aa}$ & & $0 \mathrm{Aa}$ & $100,0 \mathrm{Aa}$ \\
\hline +Sulfom & $1809+510+43,5$ & $99,7 \mathrm{Aa}$ & $100,0 \mathrm{Aa}$ & $100,0 \mathrm{Aa}$ & $100,0 \mathrm{Aa}$ & $100,0 \mathrm{Aa}$ & & $100,0 \mathrm{Aa}$ & $100,0 \mathrm{Aa}$ \\
\hline & 1190 & $99,0 \mathrm{Aa}$ & $99,3 \mathrm{Aa}$ & & $100,0 \mathrm{Aa}$ & $100,0 \mathrm{Aa}$ & & $26,7 \mathrm{Bb}$ & $100,0 \mathrm{Aa}$ \\
\hline \multirow[t]{3}{*}{ Amicarb+Isoxafl ${ }^{2}$} & $840+82,5$ & $99,0 \mathrm{Aa}$ & $100,0 \mathrm{Aa}$ & $99,3 \mathrm{Aa}$ & $100,0 \mathrm{Aa}$ & $100,0 \mathrm{Aa}$ & $100,0 \mathrm{Aa}$ & $100,0 \mathrm{Aa}$ & $100,0 \mathrm{Aa}$ \\
\hline & & C.V.(\%) & 7,5 & F trat & $10,05^{* *}$ & C.V.(\%) & 7,97 & F trat & $11,26^{* *}$ \\
\hline & & F trat x époc & $9,74^{* *}$ & F époc & $10,05^{* *}$ & F trat $x$ époc & $11,26^{* *}$ & F époc & $11,26^{* *}$ \\
\hline
\end{tabular}

Médias seguidas de mesma letra dentro de cada avaliação, maiúsculas na coluna e minúsculas na linha, não diferem entre si pelo teste de Tukey a $5 \%$. $*$ significativo a $5 \%$ de probabilidade pelo teste $\mathrm{F}$. ${ }^{*} *$ significativo a $1 \%$ de probabilidade pelo teste $\mathrm{F}$. ${ }^{1}$ diuron + hexazinone + sulfometuronmethyl, ${ }^{2}$ amicarbazone + isoxaflutole.

Dentro dos períodos de 0, 30 e $90 \mathrm{DRH}$, os tratamentos químicos não diferiram entre si para D. nuda (Tabela 2). No entanto, com 60 DRH, diuron + hexazinone + sulfometuronmethyl mostrou-se superior a partir de 14 DARU. Nesse período, o amicarbazone diferiu dos demais, proporcionando controle de $40 \%$ sobre D. nuda; seguido de amicarbazone + isoxaflutole $(58,4 \%)$ e a segunda menor dose do tratamento diuron + hexazinone + sulfometuron-methyl $(83,3 \%)$. No decorrer das avaliações, o amicarbazone foi perdendo seu efeito de controle, sendo que aos 28 DARU, proporcionou controle de apenas $16,7 \%$ isolado e 50,0\% com a adição de isoxaflutole. Aos 60 DRH a biomassa de $D$. nuda, com a mistura em 
tanque amicarbazone + isoxaflutole não diferiu da testemunha. Aos 28 DARU, com 60 DRH, diuron + hexazinone + sulfometuron-methyl foi superior ao amicarbazone e amicarbazone + isoxaflutole controlando eficientemente a $D$. nuda $(91 \%)$ a partir de sua menor dose.

Tabela 2. Controle visual (\%) de Digitaria nuda (DIGNU) submetidas a 0, 30, 60 e 90 dias de restrição hídrica (DRH), aos 7, 14, 21 e 28 dias após o restabelecimento da umidade (DARU). Jaboticabal, SP. 2011.

\begin{tabular}{|c|c|c|c|c|c|c|c|c|c|}
\hline \multirow{2}{*}{ Tratamentos } & \multirow{2}{*}{ Dose (g i.a. ha-1) } & \multicolumn{4}{|c|}{ Controle de DIGNU aos 7 DARU } & \multicolumn{4}{|c|}{ Controle de DIGNU aos 14 DARU } \\
\hline & & $0 \mathrm{DRH}$ & $30 \mathrm{DRH}$ & $60 \mathrm{DRH}$ & $90 \mathrm{DRH}$ & $0 \mathrm{DRH}$ & $30 \mathrm{DRH}$ & $60 \mathrm{DRH}$ & $90 \mathrm{DRH}$ \\
\hline $\mathrm{Diu}+\mathrm{Hex}+$ Sulfom ${ }^{1}$ & $387+391+33,35$ & $60,0 \mathrm{Aa}$ & $86,7 \mathrm{Aa}$ & $85,0 \mathrm{Aa}$ & $83,3 \mathrm{Aa}$ & $100,0 \mathrm{Aa}$ & $80,0 \mathrm{Aa}$ & $86,7 \mathrm{Aa}$ & $88,3 \mathrm{Aa}$ \\
\hline $\mathrm{Diu}+\mathrm{Hex}+$ & $1507,5+425+36,25$ &, $0 \mathrm{Aab}$ & $6,7 \mathrm{Aa}$ & $20,0 \mathrm{Bb}$ & $96,7 \mathrm{Aa}$ & $99,3 \mathrm{Aa}$ & $82,3 \mathrm{Aa}$ & $83,3 \mathrm{Aba}$ & $85,0 \mathrm{Aa}$ \\
\hline $\mathrm{Diu}+\mathrm{Hex}+$ & $1658,25+467,5+39,87$ & $41,7 \mathrm{Aa}$ & $86,7 \mathrm{Aa}$ & $65,0 \mathrm{ABa}$ & $91,7 \mathrm{Aa}$ & $99,7 \mathrm{Aa}$ & $94,0 \mathrm{Aa}$ & $98,3 \mathrm{Aa}$ & $93,3 \mathrm{Aa}$ \\
\hline $\mathrm{Diu}+\mathrm{Hex}$ & $1809+510+43,5$ & $60,0 \mathrm{Aa}$ & $5,7 \mathrm{Aa}$ & $46,7 \mathrm{ABa}$ & $83,3 \mathrm{Aa}$ & $100,0 \mathrm{Aa}$ & $85,0 \mathrm{Aa}$ & $96,7 \mathrm{Aa}$ & $88,3 \mathrm{Aa}$ \\
\hline Amicarbazone & 1190 & $40,0 \mathrm{Aa}$ & $96,7 \mathrm{Aa}$ & $36,7 \mathrm{ABa}$ & $86,7 \mathrm{Aa}$ & $100,0 \mathrm{Aa}$ & $80,0 \mathrm{Aab}$ & $40,0 \mathrm{Bb}$ & $61,7 \mathrm{Aab}$ \\
\hline \multirow[t]{3}{*}{ Amicarb+Isoxafl ${ }^{2}$} & $840+82,5$ & $43,3 \mathrm{Aa}$ & $86,7 \mathrm{Aa}$ & $58,3 \mathrm{ABa}$ & $86,7 \mathrm{Aa}$ & $100,0 \mathrm{Aa}$ & $98,3 \mathrm{Aab}$ & $58,3 \mathrm{Abb}$ & $80,0 \mathrm{Aab}$ \\
\hline & & C.V. $(\%)$ & 38,7 & F trat & $0,52 \mathrm{~ns}$ & C.V. $(\%)$ & 21,75 & F trat & $2,72^{*}$ \\
\hline & & F trat $x$ époc & $0,76 \mathrm{~ns}$ & F époc & $11,52 * *$ & F trat $x$ époc & $1,13 \mathrm{~ns}$ & F époc & $4,70 * *$ \\
\hline \multirow{2}{*}{ Tratamentos } & \multirow{2}{*}{ Dose (g i.a. ha $\left.{ }^{-1}\right)$} & \multicolumn{4}{|c|}{ Controle de DIGNU aos 21 DARU } & \multicolumn{4}{|c|}{ Controle de DIGNU aos 28 DARU } \\
\hline & & $0 \mathrm{DRH}$ & $30 \mathrm{DRH}$ & $60 \mathrm{DRH}$ & $90 \mathrm{DRH}$ & $0 \mathrm{DRH}$ & $30 \mathrm{DRH}$ & $60 \mathrm{DRH}$ & $90 \mathrm{DRH}$ \\
\hline $\mathrm{Diu}+\mathrm{Hex}+\mathrm{S}$ & & $100,0 \mathrm{Aa}$ & $99,0 \mathrm{Aa}$ & $96,7 \mathrm{Aa}$ & $100,0 \mathrm{Aa}$ & $100,0 \mathrm{Aa}$ & $100,0 \mathrm{Aa}$ & $91,7 \mathrm{Aa}$ & $94,3 \mathrm{Aa}$ \\
\hline & $1507,5+42$ & $0,0 \mathrm{Aa}$ & $0,0 \mathrm{Aa}$ & $91,7 \mathrm{Aa}$ & $0,0 \mathrm{Aa}$ & $100,0 \mathrm{Aa}$ & $0 \mathrm{Aa}$ & $100,0 \mathrm{Aa}$ & $95,3 \mathrm{Aa}$ \\
\hline -Hex+Sulfom & $1658,25+467,5+39,87$ & $100,0 \mathrm{Aa}$ & $100,0 \mathrm{Aa}$ & $99,7 \mathrm{Aa}$ & $100,0 \mathrm{Aa}$ & $100,0 \mathrm{Aa}$ & $100,0 \mathrm{Aa}$ & $100,0 \mathrm{Aa}$ & $98,7 \mathrm{Aa}$ \\
\hline -Hex+Sulfom & $1809+510+43,5$ & $100,0 \mathrm{Aa}$ & $99,7 \mathrm{Aa}$ & $99,3 \mathrm{Aa}$ & $98,3 \mathrm{Aa}$ & $100,0 \mathrm{Aa}$ & $100,0 \mathrm{Aa}$ & $100,0 \mathrm{Aa}$ & $96,0 \mathrm{Aa}$ \\
\hline rbazone & 1190 & $0,0 \mathrm{Aa}$ & $100,0 \mathrm{Aa}$ & $30,0 \mathrm{Bb}$ & $86,7 \mathrm{Aa}$ & $100,0 \mathrm{Aa}$ & $100,0 \mathrm{Aa}$ & $16,7 \mathrm{Cb}$ & $86,7 \mathrm{Aa}$ \\
\hline \multirow[t]{3}{*}{ Amicarb+Isoxafl ${ }^{2}$} & $840+82,5$ & $100,0 \mathrm{Aa}$ & $100,0 \mathrm{Aa}$ & $81,7 \mathrm{Aa}$ & $93,3 \mathrm{Aa}$ & $100,0 \mathrm{Aa}$ & $100,0 \mathrm{Aa}$ & $50,0 \mathrm{Bb}$ & $92,3 \mathrm{Aa}$ \\
\hline & & C.V.(\%) & 13,58 & F trat & $4,61 * *$ & C.V. $(\%)$ & 12,55 & F trat & $8,46^{* *}$ \\
\hline & & F trat $x$ époc & $3,00 * *$ & F époc & $6,86^{* * *}$ & F trat $x$ époc & $6,45^{* * *}$ & F époc & $16,63^{* *}$ \\
\hline
\end{tabular}

Médias seguidas de mesma letra dentro de cada avaliação, maiúsculas na coluna e minúsculas na linha, não diferem entre si pelo teste de Tukey a $5 \%$. * significativo a $5 \%$ de probabilidade pelo teste $\mathrm{F}$. ** significativo a $1 \%$ de probabilidade pelo teste $\mathrm{F}$. ${ }^{1}$ diuron + hexazinone + sulfometuronmethyl, ${ }^{2}$ amicarbazone + isoxaflutole.

Assim como D. nuda, para o controle de $P$. maximum os tratamentos químicos só diferiram entre si com 60 DRH (Tabela 3). As diuron + hexazinone + isoxaflutole, nas doses de $1658,25+467,5+39,87$ e $1809+510+$ 43,5 g i.a. ha ${ }^{-1}$, mantiveram controle total nas últimas avaliações. Amicarbazone + isoxaflutole apresentou o menor controle aos 14 DARU, diferindo dos demais tratamentos. Entretanto, ao final dos 28 DARU, atingiu nível de controle satisfatório $(83,3 \%)$. Na última avaliação, o amicarbazone isolado diferiu dos demais, proporcionando controle insatisfatório de $P$. maximum $(46,7 \%)$ e biomassa de $35,8 \%$, em relação à testemunha.

A restrição do amicarbazone a longos períodos de déficit hídrico, citado por Rodrigues e Almeida (2011), foi confirmada com o presente estudo, uma vez que o período de sessenta dias de restrição hídrica influenciou sobremaneira na eficiência do produto (Tabela 4).

Por outro lado, os resultados se contrapõem aos de Carbonari et al. (2009), em que eficazes níveis de controle de Digitaria spp. por amicarbazone foram reportados em diferentes condições de umidade do solo. No presente estudo, a $D$. nuda foi controlada por amicarbazone em condição de umidade, porém o controle não foi satisfatório quando houve 60 dias se restrição hídrica. Esse resultado contraditório entre os dois estudos pode ser explicado pela espécie de Digitaria utilizada; uma vez que a espécie Digitaria nuda é conhecida por ser a de mais difícil controle (Dias et al., 2009). Para Brachiaria plantaginea, Carbonari et al. (2009) encontraram boa eficiência de controle na condição de seca, mas ainda foi inferior ao resultado na ocorrência de chuvas. 
Tabela 3. Controle visual (\%) de Panicum maximum (PANMA), submetidas a 0, 30, 60 e 90 dias de restrição hídrica (DRH), aos 7, 14, 21 e 28 dias após o restabelecimento da umidade (DARU). Jaboticabal, SP. 2011.

\begin{tabular}{|c|c|c|c|c|c|c|c|c|}
\hline \multirow{2}{*}{ Tratamentos } & \multirow{2}{*}{ Dose (g i.a. ha-1) } & \multicolumn{4}{|c|}{ Controle de PANMA aos 7 DARU } & \multicolumn{3}{|c|}{ Controle de PANMA aos 14 DARU } \\
\hline & & $0 \mathrm{DRH}$ & $30 \mathrm{DRH}$ & $60 \mathrm{DRH}$ & $90 \mathrm{DRH}$ & $0 \mathrm{DRH}$ & $30 \mathrm{DRH} \quad 60 \mathrm{DRH}$ & $90 \mathrm{DRH}$ \\
\hline Diu + Hex + Sulfom ${ }^{1}$ & $387+391+33,35$ & $71,7 \mathrm{Aa}$ & $48,3 \mathrm{Aa}$ & $73,3 \mathrm{Aa}$ & $52,7 \mathrm{Aa}$ & $98,3 \mathrm{Aa}$ & $100,0 \mathrm{Aa} 86,7 \mathrm{Ab}$ & $100,0 \mathrm{Aa}$ \\
\hline Diu+Hex+Sulfom & $1507,5+425+36,25$ & $70,0 \mathrm{Aa}$ & $65,0 \mathrm{Aa}$ & $31,7 \mathrm{Aa}$ & $69,0 \mathrm{Aa}$ & $99,0 \mathrm{Aa}$ & $100,0 \mathrm{Aa} \quad 95,0 \mathrm{Aa}$ & $100,0 \mathrm{Aa}$ \\
\hline Diu + Hex + Sulfom & $1658,25+467,5+39,87$ & $63,3 \mathrm{Aa}$ & $35,0 \mathrm{Aa}$ & $45,0 \mathrm{Aa}$ & $51,7 \mathrm{Aa}$ & $100,0 \mathrm{Aa}$ & $100,0 \mathrm{Aa} 95,0 \mathrm{Aa}$ & $100,0 \mathrm{Aa}$ \\
\hline $\mathrm{Diu}+\mathrm{Hex}+$ & $1809+510+43,5$ & $63,3 \mathrm{Aa}$ & $46,7 \mathrm{Aa}$ & $53,3 \mathrm{Aa}$ & $63,3 \mathrm{Aa}$ & $100,0 \mathrm{Aa}$ & $100,0 \mathrm{Aa} 96,7 \mathrm{Aa}$ & $100,0 \mathrm{Aa}$ \\
\hline Amicarbaz & 1190 & $45,0 \mathrm{Aa}$ & $50,0 \mathrm{Aa}$ & $26,7 \mathrm{Aa}$ & $43,3 \mathrm{Aa}$ & $98,3 \mathrm{Aab}$ & $100,0 \mathrm{Aa} \quad 90,0 \mathrm{Ab}$ & $100,0 \mathrm{Aa}$ \\
\hline \multirow[t]{3}{*}{ Amicarb+Isoxafl ${ }^{2}$} & $840+82,5$ & $30,0 \mathrm{Aa}$ & $73,3 \mathrm{Aa}$ & $61,7 \mathrm{Aa}$ & $70,0 \mathrm{Aa}$ & $100,0 \mathrm{Aa}$ & $100,0 \mathrm{Aa} \quad 75,0 \mathrm{Bb}$ & $100,0 \mathrm{Aa}$ \\
\hline & & C.V. $(\%)$ & 48,06 & F trat. & $1,06 \mathrm{~ns}$ & C.V.(\%) & $4,63 \quad$ F trat. & $2,46^{*}$ \\
\hline & & F trat $x$ époc & $0,95 \mathrm{~ns}$ & F époc. & $0,52 \mathrm{~ns}$ & F trat $x$ époc & F époc. & $22,47 * *$ \\
\hline \multirow{2}{*}{ Tratamentos } & \multirow{2}{*}{ Dose (g i.a. ha-1) } & \multicolumn{4}{|c|}{ Controle de PANMA aos 21 DARU } & \multicolumn{3}{|c|}{ Controle de PANMA aos 28 DARU } \\
\hline & & $0 \mathrm{DRH}$ & $30 \mathrm{DRH}$ & $60 \mathrm{DRH}$ & $90 \mathrm{DRH}$ & $0 \mathrm{DRH}$ & $30 \mathrm{DRH} \quad 60 \mathrm{DRH}$ & $90 \mathrm{DRH}$ \\
\hline $\mathrm{Diu}+\mathrm{Hex}+$ Sulfom ${ }^{1}$ & $387+391+33,35$ & $100,0 \mathrm{Aa}$ & $100,0 \mathrm{Aa}$ & $88,3 \mathrm{Aa}$ & $100,0 \mathrm{Aa}$ & $100,0 \mathrm{Aa}$ & $100,0 \mathrm{Aa} \quad 100,0 \mathrm{Aa}$ &, $0 \mathrm{Aa}$ \\
\hline $\mathrm{Diu}+\mathrm{Hex}^{+}$ & $1507,5+42$ & $100,0 \mathrm{Aa}$ & $0 \mathrm{Aa}$ & $97,3 \mathrm{Aa}$ & $100,0 \mathrm{Aa}$ & $100,0 \mathrm{Aa}$ & $\mathrm{Aa} 10$ & $0,0 \mathrm{Aa}$ \\
\hline Diu+Hex+Sulfom & $1658,25+467,5+39,87$ & $100,0 \mathrm{Aa}$ & $100,0 \mathrm{Aa}$ & $100,0 \mathrm{Aa}$ & $100,0 \mathrm{Aa}$ & $100,0 \mathrm{Aa}$ & $100,0 \mathrm{Aa} \quad 100,0 \mathrm{Aa}$ & $98,3 \mathrm{Aa}$ \\
\hline Diu+Hex +Sulfom & $1809+510+43,5$ & $100,0 \mathrm{Aa}$ & $100,0 \mathrm{Aa}$ & $100,0 \mathrm{Aa}$ & $100,0 \mathrm{Aa}$ & $100,0 \mathrm{Aa}$ & $100,0 \mathrm{Aa} 100,0 \mathrm{Aa}$ & $100,0 \mathrm{Aa}$ \\
\hline Amicarbazone & 1190 & $100,0 \mathrm{Aa}$ & $100,0 \mathrm{Aa}$ & $66,7 \mathrm{Bb}$ & $100,0 \mathrm{Aa}$ & $96,7 \mathrm{Aa}$ & $96,7 \mathrm{Aa} \quad 46,7 \mathrm{Bb}$ & $100,0 \mathrm{Aa}$ \\
\hline \multirow[t]{3}{*}{ Amicarb+Isoxafl ${ }^{2}$} & $840+82,5$ & $100,0 \mathrm{Aa}$ & $100,0 \mathrm{Aa}$ & $87,7 \mathrm{Aa}$ & $100,0 \mathrm{Aa}$ & $100,0 \mathrm{Aa}$ & $100,0 \mathrm{Aa} \quad 83,3 \mathrm{Aa}$ & $100,0 \mathrm{Aa}$ \\
\hline & & C.V. $(\%)$ & 6,81 & F trat. & $2,74^{*}$ & C.V. $(\%)$ & $12,33 \quad$ F trat. & $3,01^{*}$ \\
\hline & & F trat $x$ époc & $2,74 * *$ & F époc. & $10,19 * *$ & F trat $x$ époc & F époc. & $3,97 *$ \\
\hline
\end{tabular}

Médias seguidas de mesma letra dentro de cada avaliação, maiúsculas na coluna e minúsculas na linha, não diferem entre si pelo teste de Tukey a $5 \%$. * significativo a $5 \%$ de probabilidade pelo teste $\mathrm{F}$. $* *$ significativo a $1 \%$ de probabilidade pelo teste $\mathrm{F}$. ${ }^{1}$ diuron + hexazinone + sulfometuronmethyl, ${ }^{2}$ amicarbazone + isoxaflutole.

Tabela 4. Biomassa (mg) de B. decumbens, $D$. nuda e $P$. maximum, submetidas a $0,30,60$ e 90 dias de restrição hídrica, aos 7, 14, 21 e 28 dias após o restabelecimento da umidade. Jaboticabal, SP. 2011.

\begin{tabular}{|c|c|c|c|c|c|}
\hline \multirow{2}{*}{ Tratamentos } & \multirow{2}{*}{ Dose (g i.a. ha- $\left.{ }^{-1}\right)$} & \multicolumn{4}{|c|}{ Biomassa (mg) de BRADC } \\
\hline & & $0 \mathrm{DRH}$ & $30 \mathrm{DRH}$ & $60 \mathrm{DRH}$ & $90 \mathrm{DRH}$ \\
\hline $\begin{array}{l}\text { Diu+Hex+Sulfom }{ }^{1} \\
\text { Diu+Hex+Sulfom } \\
\text { Diu+Hex+Sulfom } \\
\text { Diu+Hex+Sulfom } \\
\text { Amicarbazone } \\
\text { Amicarb+Isoxafl } \\
\text { Testemunha } \\
\end{array}$ & $\begin{array}{c}387+391+33,35 \\
1507,5+425+36,25 \\
1658,25+467,5+39,87 \\
1809+510+43,5 \\
1190 \\
840+82,5 \\
-\end{array}$ & $\begin{array}{c}0 \mathrm{Ba} \\
0 \mathrm{Ba} \\
0 \mathrm{Ba} \\
0 \mathrm{Ba} \\
0 \mathrm{Bb} \\
0 \mathrm{Ba} \\
1233 \mathrm{Aa} \\
\end{array}$ & $\begin{array}{l}0 \mathrm{Ba} \\
0 \mathrm{Ba} \\
0 \mathrm{Ba} \\
0 \mathrm{Ba} \\
0 \mathrm{Bb} \\
0 \mathrm{Ba} \\
536 \mathrm{Ab}\end{array}$ & $\begin{array}{l}107 \mathrm{Ba} \\
0 \mathrm{Ba} \\
0 \mathrm{Ba} \\
0 \mathrm{Ba} \\
505 \mathrm{Aa} \\
27 \mathrm{Ba} \\
623 \mathrm{Ab} \\
\end{array}$ & $\begin{array}{c}0 \mathrm{Ba} \\
0 \mathrm{Ba} \\
0 \mathrm{Ba} \\
0 \mathrm{Ba} \\
231 \mathrm{Bab} \\
0 \mathrm{Ba} \\
823 \mathrm{Ab}\end{array}$ \\
\hline $\begin{array}{l}\text { C.V.(\%) } \\
\text { F trat x épocas } \\
\end{array}$ & $\begin{array}{c}132,49 \\
1,96^{*} \\
\end{array}$ & & & $\begin{array}{r}30,2 \\
1,2 \\
\end{array}$ & \\
\hline \multirow{2}{*}{ Tratamentos } & \multirow{2}{*}{ Dose (g i.a. ha ${ }^{-1}$ ) } & \multicolumn{4}{|c|}{ Biomassa (g) de DIGNU } \\
\hline & & 0DRH & $30 \mathrm{DRH}$ & $60 \mathrm{DRH}$ & $90 \mathrm{DRH}$ \\
\hline Diu + Hex + Sulfom ${ }^{1}$ & $387+391+33,35$ & $0 \mathrm{Aa}$ & $0 \mathrm{Ba}$ & $98 \mathrm{Ba}$ & $0 \mathrm{Aa}$ \\
\hline Diu+Hex+Sulfom & $1507,5+425+36,25$ & $0 \mathrm{Aa}$ & $0 \mathrm{Ba}$ & $1 \mathrm{Ba}$ & $0 \mathrm{Aa}$ \\
\hline $\mathrm{Diu}+\mathrm{Hex}+$ Sulfom & $1658,25+467,5+39,87$ & $\mathrm{OAa}$ & $0 \mathrm{Ba}$ & $0 \mathrm{Ba}$ & $\mathrm{OAa}$ \\
\hline $\mathrm{Diu}+\mathrm{Hex}+$ Sulfom & $1809+510+43,5$ & $0 \mathrm{Aa}$ & $0 \mathrm{Ba}$ & $0 \mathrm{Ba}$ & $0 \mathrm{Aa}$ \\
\hline Amicarbazone & 1190 & $0 \mathrm{Ab}$ & $0 \mathrm{Bb}$ & $303 \mathrm{Aa}$ & $19 \mathrm{Ab}$ \\
\hline Amicarb+Isoxafl ${ }^{2}$ & $840+82,5$ & $0 \mathrm{Aa}$ & $0 \mathrm{Ba}$ & $18 \mathrm{Ba}$ & $2 \mathrm{Aa}$ \\
\hline Testemunha & - & $139 \mathrm{Ab}$ & $333 \mathrm{Aa}$ & $179 \mathrm{Abab}$ & $80 \mathrm{Ab}$ \\
\hline $\begin{array}{l}\text { C.V.(\%) } \\
\text { F trat x épocas }\end{array}$ & $\begin{array}{l}173,30 \\
2,74^{* *}\end{array}$ & $\begin{array}{r}F t \\
F \text { ép }\end{array}$ & & $\begin{array}{r}10, \\
4,2\end{array}$ & \\
\hline \multirow{2}{*}{ Tratamentos } & \multirow{2}{*}{ Dose (g i.a. ha $\left.a^{-1}\right)$} & \multicolumn{4}{|c|}{ Biomassa (g) de PANMA } \\
\hline & & $0 \mathrm{DRH}$ & $30 \mathrm{DRH}$ & $60 \mathrm{DRH}$ & $90 \mathrm{DRH}$ \\
\hline $\mathrm{Diu}+\mathrm{Hex}+$ Sulfom ${ }^{1}$ & $387+391+33,35$ & $0 \mathrm{Ba}$ & $0 \mathrm{Ba}$ & $0 \mathrm{Ba}$ & $0 \mathrm{Ba}$ \\
\hline Diu+Hex+Sulfom & $1507,5+425+36,25$ & $0 \mathrm{Ba}$ & $0 \mathrm{Ba}$ & $0 \mathrm{Ba}$ & $0 \mathrm{Ba}$ \\
\hline Diu+Hex+Sulfom & $1658,25+467,5+39,87$ & $1 \mathrm{Ba}$ & $0 \mathrm{Ba}$ & $0 \mathrm{Ba}$ & $0 \mathrm{Ba}$ \\
\hline Diu+Hex+Sulfom & $1809+510+43,5$ & $0 \mathrm{Ba}$ & $0 \mathrm{Ba}$ & $0 \mathrm{Ba}$ & $0 \mathrm{Ba}$ \\
\hline Amicarbazone & 1190 & $0 \mathrm{Ba}$ & $7 \mathrm{Ba}$ & $397 \mathrm{Ba}$ & $257 \mathrm{Ba}$ \\
\hline Amicarb+Isoxafl ${ }^{2}$ & $840+82,5$ & $0 \mathrm{Ba}$ & $0 \mathrm{Ba}$ & $73 \mathrm{Ba}$ & $2 \mathrm{Ba}$ \\
\hline Testemunha & - & $562 \mathrm{Ab}$ & $1222 \mathrm{Aa}$ & $1108 \mathrm{Aa}$ & $1160 \mathrm{Aa}$ \\
\hline C.V.(\%) & 118,26 & \multicolumn{2}{|c|}{$\mathrm{F}$ trat } & \multicolumn{2}{|c|}{$41,49^{* *}$} \\
\hline $\mathrm{F}$ trat $\mathrm{x}$ épocas & $1,26 \mathrm{~ns}$ & \multicolumn{2}{|c|}{ F épocas } & \multicolumn{2}{|c|}{$2,09 \mathrm{~ns}$} \\
\hline
\end{tabular}

Médias seguidas de mesma letra dentro de cada avaliação, maiúsculas na coluna e minúsculas na linha, não diferem entre si pelo teste de Tukey a $5 \%$. * significativo a $5 \%$ de probabilidade pelo teste $\mathrm{F}$. ** significativo a $1 \%$ de probabilidade pelo teste $\mathrm{F}$. ' diuron + hexazinone + sulfometuron-methyl, ${ }^{2}$ amicarbazone + isoxaflutole.
Os resultados comprovam o excelente controle das gramíneas proporcionado pela associação diuron + hexazinone + sulfometuron-methyl, que após 28 DARU, foi altamente eficaz para o controle das três espécies. Em estudo em que as folhas largas foram o alvo, a associação diuron + hexazinone foi suficiente para o controle das plantas daninhas (Toledo et al., 2010). O diuron possui relativa persistência nos solos e o hexazinone considerável solubilidade (Rodrigues \& Almeida, 2011), características que, somadas às do sulfometuron-methyl, podem ter conferido à mistura o excelente resultado observado. Um possível efeito sinérgico entre as moléculas pode ter ocorrido, porém ainda são necessários novos estudos para comprovar a hipótese, como a utilização da aplicação de cada ingrediente ativo isoladamente.

\section{Conclusões}

$\mathrm{O}$ herbicida diuron + hexazinone + sulfometuron-methyl, nas doses de $1387+391$ $+33,35 ; 1507,5+425+36,25 ; 1658,25+$ $467,5+39,87$ e $1809+510+43,5$ g i.a. ha $^{-1}$, 
foi eficaz no controle de Brachiaria decumbens, Digitaria nuda e Panicum maximum em períodos de restrição hídrica, apresentando resultados similares à associação de amicarbazone + isoxaflutole $(840+82,5 \mathrm{~g}$ i.a. ha $\left.^{-1}\right)$ e superiores a amicarbazone $(1190 \mathrm{~g}$ i.a. ha $^{-1}$ ), quando aplicado sob condição de 60 dias de seca.

\section{Referências}

CARBONARI, C.A. et al. Efeitos de diferentes condições de umidade do solo e profundidades de germinação de Brachiaria plantaginea e Digitaria spp. sobre a eficácia do herbicida amicarbazone. Revista Brasileira de Herbicidas, v.8, n.3, p.68-74, 2009.

CORREIA, N.M.; BRAZ, B.A.; FUZITA, W.E. Eficácia de herbicidas aplicados nas épocas seca e úmida para o controle de Merremia aegyptia na cultura da cana-deaçúcar. Planta Daninha, v.28, n.3, p.631-642, 2010.

DIAS, A.C.R.; NICOLAI, M.; CHRISTOFFOLETI, P.J. Capim-colchão Identificação e manejo na cultura da canade-açúcar. Coordenação: CHRISTOFFOLETI, P. J. Piracicaba: USP/ESALQ, 2009, 60 p.

KRUSE, N.D. et al. Sinergismo potencial entre herbicidas inibidores do fotossistema II e da síntese de carotenóides. Ciência Rural, v.31, n.4, p.569-575, 2001.

MARTINI, G.; DURIGAN, J.C. Influência do teor de água na superfície do solo sobre a eficácia e seletividade do flazasulfuron, na cultura de cana-de-açúcar. Planta Daninha, v.22, n.2, p.259-267, 2004.

MATTHEWS, J.M. Management of herbicide resistant weed populations. In: POWLES, S.; HOLTUM, J. (eds.) Herbicide resistance in plants: biology and biochemistry. Boca Raton: CRC, 1994. p. 317-335.

NEGRISOLI, E. et al. Influência da palha e da simulação de chuva sobre a eficácia da mistura formulada clomazone + hexazinone no controle de plantas daninhas em área de cana-crua. Planta Daninha, v.29, n.1, p.169-177, 2011.

PROCÓPIO, S.O.; SILVA, A.A.; VARGAS, L. Manejo e controle de plantas daninhas em cana-de-açúcar. In.: VARGAS, L.; ROMAN, E. S. (Eds.). Manual de manejo e controle de plantas daninhas. Bento Gonçalves: Embrapa Uva e Vinho, 2004. p. 397-452.

RODRIGUES, B.N.; ALMEIDA, F.S. Guia de herbicidas. $6^{\mathrm{a}}$ ed., Londrina, 2011. 697p.

SILVA, A.A. et al. Controle de plantas daninhas. Brasília: Associação Brasileira de Educação Agrícola Superior; Viçosa, MG: Universidade Federal de Viçosa, 1999. 260 p.

TOLEDO, R.E.B. et al. Dinamic (Amicarbazone), novo herbicida seletivo para o controle de plantas daninhas em pré e pós emergência na cultura da cana-de-açúcar. In: CONGRESSO BRASILEIRO DA CIÊNCIA DAS PLANTAS DANINHAS, 24., 2004, São Pedro. Resumos... São Pedro: 2004. 245 p.

TOLEDO, R.E.B. et al. Manejo de corda-deviola (Ipomoea grandifolia) no sistema de cana-crua com herbicidas Front, Velpar K e associações de Velpar K e outros herbicidas. In: XXVII CONGRESSO BRASILEIRO DA CIÊNCIA DAS PLANTAS DANINHAS. Anais... Ribeirão Preto, 2010. p 2407-2409. 